\title{
Experimental Evaluation of the Depth Resolution of a Raman Microscope
}

\author{
F. Adar,* E. Lee,* S. Mamedov,* and A. Whitley* \\ * Molecular Microanalysis Division, HORIBA Scientific, 3880 Park Ave., Edison NJ \\ 08820
}

Raman microscopy has been attractive because of its ability to characterize materials on a spatial scale commensurate with optical microscopy. Typically the lateral spatial resolution is quoted as determined by the Airy disc[1] which is $1.22 \lambda / \mathrm{NA}$ where $\lambda$ is the wavelength of the illuminating light, and NA is the numerical aperture which is equal to nsin $\theta$, where $n$ is the index of refraction of the medium (1.0 in the case of air) and $\square$ is the angle subtended by the optics. However, the Airy disc description cannot be correct for a Raman microscope. The Airy disc assumes uniform illumination of the focusing optic, and the laser profile is anything but. In addition, in some instruments the Gaussian laser profile is not well matched to the aperture of the focusing objective. At any rate, this article is going to concentrate on the depth resolution of the Raman microscope. Optical calculations for depth resolution of an optical microscope state that the it is proportional to $\lambda /(\mathrm{NA})^{2}$. The essential point to recognize is that the spatial resolution of any Raman microscope depends on the detection optics as well as the focusing optics. How effectively does the optical system collect the Raman signal excited in the laser focal spot, and reject the signal from the surrounding volume that is illuminated by the laser but not in focus?

Then the essential question becomes how to evaluate the depth resolution experimentally. Historically people have used a piece of a polished Si wafer for these tests. But this material was originally chosen more for the repeatability of any measurement of its signal rather than its appropriateness for answering the questions of depth resolution. The problem with using silicon is that when performing a depth profile, as the sample surface is moved away from the focal plane, the laser-illuminated area is increased; the final signal is a convolution of the losses because the laser illuminated area is not passed efficiently through the confocal hole, and the increase in signal because of the increased excitation volume. In addition, the depth of penetration of the laser into the crystal is not necessarily negligible. At $633 \mathrm{~nm}$, it will penetrate $3 \mu \mathrm{m}$, at $785 \mathrm{~nm} 12 \mu \mathrm{m}$. In trying to determine a better way to determine the confocal properties of a Raman system microscopic polymer beads were selected. With such a sample, when the sample is defocused, the Raman volume cannot be larger than the volume of the bead. Comparison of depth profiles of $2 \mu \mathrm{m}$ and $0.5 \mu \mathrm{m}$ beads and silicon will be shown to provide insight into the confocal behavior of the Raman microscope. These measurements are done using the $532 \mathrm{~nm}$ excitation wavelength whose depth of penetration into silicon is about $0.7 \mu \mathrm{m}$. This avoids the complications of volume effects.

Figure 1 shows depth profiles of the $2 \mu \mathrm{m}$ and $0.5 \mu \mathrm{m}$ spheres of polystyrene recorded while varying the confocal hole. As the confocal hole is increased, the signal strength increased because light from more of the bead volume is transmitted. However, the full 
width at half maximum (FWHM) of the profile of the $2 \mu \mathrm{m}$ bead changes only from 4.9 to $5.1 \mu \mathrm{m}$ whereas the profile of the $0.5 \mu \mathrm{m}$ bead changes from just under 2 to somewhat over $3 \mu \mathrm{m}$ as the hole is opened. From this one can conclude that the limiting depth resolution is just smaller than $2 \mu \mathrm{m}$.

Figure 2 shows a depth profile of silicon, recorded with a $25 \mu \mathrm{m}$ confocal hole, measured on bare silicon, and on silicon under one of the $0.5 \mu \mathrm{m}$ polymer spheres. Under the sphere the silicon signal was higher, and its depth profile was sharper, than from the bare silicon. The conclusion to be drawn is that the polymer sphere is acting like a solid immersion lens.

[1]. M. Born and E. Wolf, Principle of Optics: Electromagnetic Theory of Propagation, Interference and Diffraction of Light, $7^{\text {th }}$ Edition, Cambridge University Press 1999
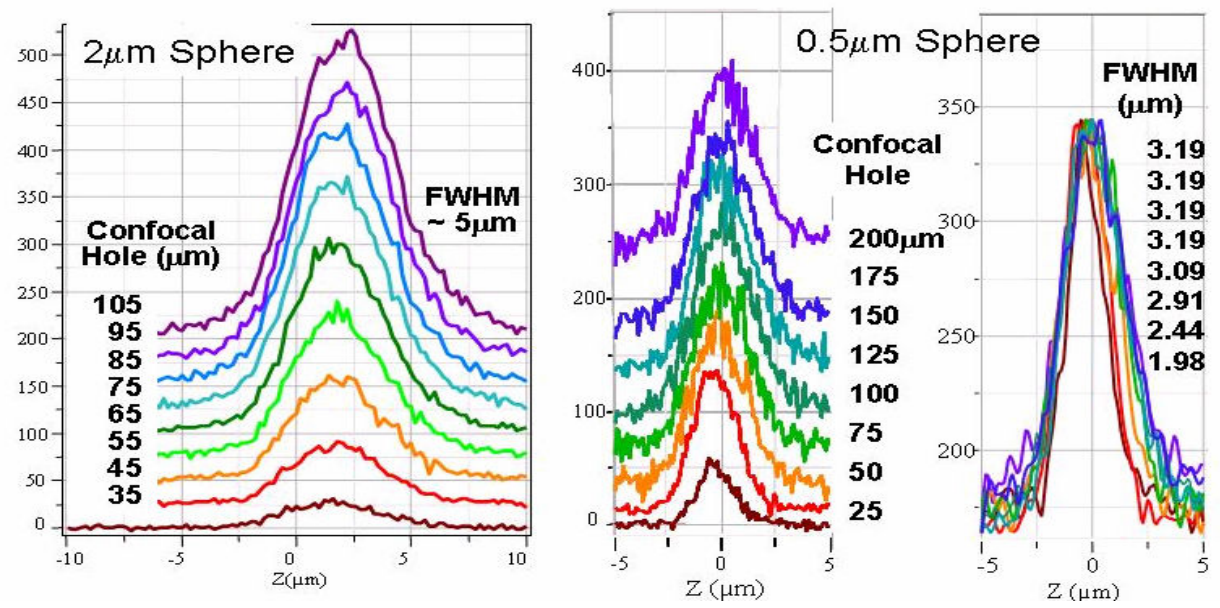

Figure 1. Confocal hole size dependence of Raman depth profiles of a $2 \mu \mathrm{m}$ sphere (left) and a $0.5 \mu \mathrm{m}$ sphere (middle and right). For the larger sphere (left), the FWHM did not vary much with the hole size, even though the signal did increase. For the smaller sphere the depth profiles are displayed to show how the signal increased with hole size (middle) and how the FWHM of the profile depended on the hole size(right).

Figure 2. Raman depth profile of $\mathrm{Si}$, recorded with $25 \mathrm{~mm}$ confocal hole, under $0.5 \mathrm{~mm}$ sphere of polymer (left trace) vs. bare silicon (right trace).

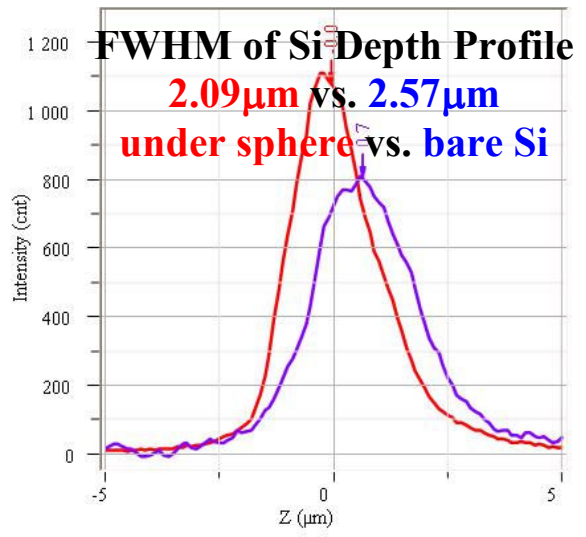

